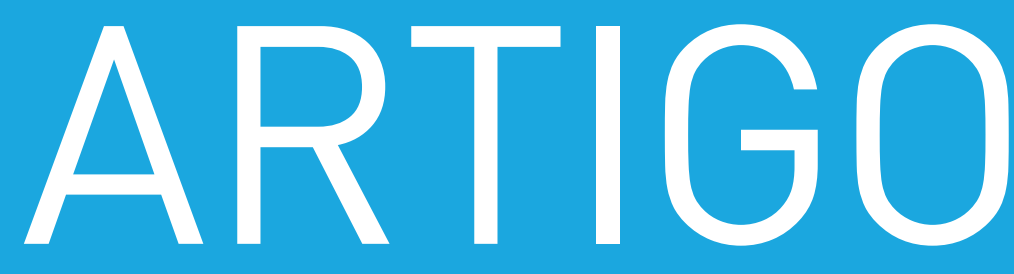

\title{
FOBIA SOCIAL EM EȘTUDANTES DE MEDICINA SUBMETIDOS AO MÉTODO DE ENSINO- APRENDIZAGEM BASEADA EM PROBLEMAS NO ESTADO DE SERGIPE
}

\section{SOCIAL PHOBIA IN MEDICAL STUDENTS SUBJECTED TO THE PROBLEM-BASED LEARNING TEACHING METHOD IN THE STATE OF SERGIPE}

\section{Resumo}

Objetivo: Identificar a prevalência de fobia social e das principais situações sociais que a caracterizam e que cursam com sintomas de ansiedade, medo ou evitação em estudantes de medicina submetidos à metodologia de ensino aprendizagem baseada em problemas (ABP) no estado de Sergipe.

Métodos: Trata-se de um recorte de um estudo clínico não experimental, exploratório, transversal, descritivo e de abordagem analítica quantitativa. Obtivemos uma amostra de 323 alunos, sendo o nosso universo todos os estudantes de medicina do estado de Sergipe cuja instituição de ensino adote o método de ABP. Para avaliação dos alunos, utilizamos um questionário específico e a Escala de Fobia Social de Liebowitz.

Resultados: A fobia social foi detectada em 30,7\% dos estudantes, com maior prevalência para a gravidade moderada. Dentro do perfil epidemiológico, o sexo feminino, os solteiros e os alunos com idade entre $18 \mathrm{e}$ 25 anos foram aqueles com maior prevalência da doença. As principais situações sociais temidas no que se refere a medo ou ansiedade foram expressar discordância ou desaprovação para alguém e o de falar com alguém que não conhece. As situações sociais mais frequentemente evitadas foram expressar discordância ou desaprovação, falar com pessoas desconhecidas e ser o centro das atenções.

Conclusão: A fobia social teve uma alta prevalência entre a amostra de universitários do curso de medicina submetidos à metodologia ABP, em Sergipe, com maior destaque para a gravidade moderada do transtorno. Com este estudo, pretendemos colocar o tema em evidência e incentivar novos trabalhos sobre o mesmo.

Palavras-chave: Fobia social, estudantes de medicina, aprendizagem baseada em problemas.

\section{Abstract}

Objective: To identify the prevalence of social phobia and the social situations that trigger it and cooccur with symptoms of anxiety, fear, or avoidance in medical students subjected to the problem-based learning (PBL) teaching methodology in the state of Sergipe.

Methods: This article is part of a non-experimental, exploratory, cross-sectional, descriptive and quantitative analytical study. We obtained a sample of 323 students, representative of all medical students in the state of Sergipe attending teaching institutions that adopt the PBL method. Students were evaluated using a specific questionnaire and the Liebowitz Social Anxiety Scale.

Results: Social phobia was detected in $30.7 \%$ of the students, with a higher prevalence of moderate severity. With regard to the epidemiological profile, female sex, being unmarried and being 18-25 years old were the characteristics associated with the highest prevalence results. The social situations most commonly feared (fear or anxiety) were expressing disagreement with or disapproval of someone and talking to someone they do 


\section{MAYANA LULA ANDRADE', KAMILA MARIA DE ANDRADE SANTOS SILVEIRA', EUSÉBIO LINO DOS SANTOS JÚNIOR², PAULO MILHOMEM FERRO NETO³ , DEBORAH PIMENTEL ${ }^{4}$}

Acadêmica de Medicina, Universidade Tiradentes, Aracaju, SE. ${ }^{2}$ Acadêmico de Medicina, Universidade Tiradentes, Aracaju, SE. ${ }^{3}$ Graduado em Medicina, Universidade Federal de Sergipe (UFS), Lagarto, SE. ${ }^{4}$ Doutora. Professora, Universidade Tiradentes, Aracaju, SE. Professora, UFS, São Cristóvão, SE.

not know. The social situations most frequently avoided were expressing disagreement or disapproval, talking to unknown people and being the center of attention.

Conclusion: Social phobia showed a high prevalence in this sample of undergraduate medical students subjected to the PBL methodology in Sergipe; the moderate severity observed should be highlighted. With this study we intend to shed light on the topic and encourage new studies to be undertaken.

Keywords: Social phobia, medical students, problembased learning.

\section{INTRODUÇÃO}

O desenvolvimento das habilidades sociais é imprescindível para o estudante de medicina, tanto no desempenho de suas atividades acadêmicas como para encarar a rotina de sua profissão futura, pautada na comunicação interpessoal e na relação médicopaciente.

Os transtornos de ansiedade, por sua vez, são as doenças mentais mais comuns na população geral. Entre eles, destaca-se a fobia social (FS), também chamada de transtorno de ansiedade social (TAS), que acontece quando o indivíduo apresenta medo ou ansiedade excessiva, irracional e persistente, mediante uma possível avaliação negativa em situações de interação social ou no desempenho1-4. Esse medo leva ao prejuízo na qualidade de vida do indivíduo e no comprometimento significativo das suas relações sociais, laborais e acadêmicas ${ }^{5-9}$. Desta forma, o indivíduo pode isolar-se socialmente e até mesmo desistir do curso de graduação, por exemplo $4,10-13$.

Nos cursos que utilizam a metodologia problem based learning (PBL), que no português significa aprendizagem baseada em problemas (ABP), essas habilidades sociais passam a ser ainda mais exigidas, pois o método preconiza a participação ativa dos alunos na construção do conhecimento. O cerne da metodologia ABP são as sessões tutoriais, em que os alunos são apresentados a uma situação-problema e precisam discuti-la em pequenos grupos, sob a supervisão e avaliação de um tutor. Em um primeiro momento, essa discussão se faz com os conhecimentos prévios dos alunos, e, em seguida, os mesmos são instruídos a traçar objetivos de estudo para, posteriormente, discutir o problema a partir de conhecimentos adquiridos na literatura. Ademais, o estudante passa a ter contato com os pacientes já no início da graduação, podendo praticar os seus conhecimentos teóricos. Através dessa metodologia, os alunos são avaliados constantemente, como na apresentação de seminários e casos clínicos, nos debates em grupo e nas sessões tutoriais ${ }^{14,15}$, o que remete a uma maior probabilidade de desenvolver alguma gravidade de FS.

Os sintomas que melhor caracterizam a FS são o medo ou ansiedade em situações sociais temidas, assim como a evitação das mesmas. No Brasil, poucos estudos se propuseram a investigá-los em universitários, e mais especificamente, no curso de medicina ${ }^{16,17}$. $\bigcirc$ objetivo deste estudo é identificar a prevalência de FS e das principais situações sociais que a caracterizam e que cursam com sintomas de ansiedade, medo ou evitação em estudantes de medicina submetidos à metodologia de ensino ABP, no estado de Sergipe.

\section{Metodologia}

Trata-se de um recorte de um estudo clínico não experimental, exploratório, transversal, descritivo e de abordagem analítica quantitativa. Nosso universo são todos os estudantes de medicina do estado de Sergipe, cuja instituição de ensino adote o método ABP. A amostra de 323 alunos teve seu cálculo baseado na fórmula de Gill18, para populações finitas, que analisa o tamanho necessário da mesma, considerando 1.600 alunos no total matriculados nessas etapas (aproximadamente 800 em cada escola/universidade), com erro amostral de 3,2\% e com um nível de confiança de 95\%.

Os critérios de inclusão foram estudantes acima de 18 anos de ambos os sexos, que assinaram o termo de consentimento livre e esclarecido (TCLE), de acordo com as normas do Conselho Nacional de Saúde do Ministério da Saúde explicitadas na resolução n 466/12, e o trabalho somente foi iniciado após aprovação do Comitê de Ética em Pesquisa da Universidade Tiradentes (CEP/UNIT), com o parecer CAAE n 67748317.4.0000.5371.

Os dados foram coletados no campus Farolândia da UNIT em Aracaju (SE) e no campus Prof. Dr. Antônio Garcia Filho da Universidade Federal de Sergipe em Lagarto (SE). Foi apresentado e explicado o projeto de pesquisa aos alunos e esclarecido o sigilo de dados, sem haver a necessidade de identificação no questionário construído especialmente para esta pesquisa, bastando, 


\section{ARTIGO ORIGINAL}

\section{MAYANA LULA ANDRADE \\ KAMILA MARIA DE ANDRADE SANTOS SILVEIRA EUSÉBIO LINO DOS SANTOS JÚNIOR PAULO MILHOMEM FERRO NETO DEBORAH PIMENTEL}

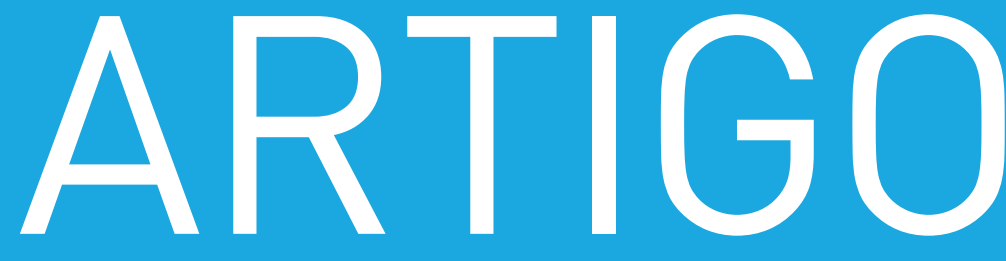

apenas, o preenchimento do mesmo e assinatura do TCLE.

Os instrumentos utilizados foram a Escala de Fobia Social de Liebowitz ${ }^{19}$ (Liebowitz Social Anxiety Scale LSAS) adaptada e um questionário construído de forma específica para a presente pesquisa. A LSAS avalia situações de interação social e de desempenho que os estudantes com FS tendem a evitar ou apresentar medo e ansiedade. É composta por 24 itens divididos em situações de interação social (11 itens) e de desempenho (13 itens) que são classificados de 0 a 3, em termos de ansiedade e/ou medo que provoca e a frequência do seu evitamento. O total de pontos é a medida da intensidade da FS que pode ser leve, moderada, grave ou muito grave.

O questionário específico tem ênfase na abordagem do perfil do estudante com FS e análise do seu desempenho acadêmico. É composto por perguntas sobre idade, sexo, estado civil, faculdade em que o aluno estuda e respectivo ano em que se encontra, se já realizou outro curso superior, se já sentiu vergonha pelo seu desempenho, se perdeu aula e faltou provas por medo, ansiedade ou evitação, se já pensou em desistir do curso e se acredita ser portador de algum transtorno psíquico.

E, por fim, após a aplicação do questionário e da LSAS $^{19}$, os achados foram inseridos em planilhas eletrônicas, e as variáveis foram descritas por meio de frequência absoluta e relativa percentual. As associações entre variáveis foram testadas por meio do teste qui-quadrado de Pearson. O nível de significância adotado foi de 5\%, o software utilizado foi o R Core Team 2018, e para todas as análises dados ausentes não foram considerados.

Para efeito deste recorte, os autores trabalharam na identificação da prevalência da FS e do medo e evitação diante de situações sociais em alunos desta amostra.

\section{Resultados}

Um total de 323 estudantes de medicina participou desta pesquisa, entre os quais 30,7\% ( $n=92)$ apresentaram o diagnóstico de FS, com maior prevalência para a gravidade moderada, com $12,7 \%(n=38)$, seguida da gravidade leve, com $11,4 \%(n=34)$, e por fim grave e muito grave, cada uma com 3,3\% ( $n=10)$ (Tabela 1).
Tabela 1 - Prevalência do transtorno de ansiedade social e seus graus em Sergipe

\begin{tabular}{lc}
\hline Transtorno de ansiedade social & Prevalência, $\mathbf{n}$ (\%) \\
\hline Sem & $231(69,2)$ \\
Leve & $34(11,4)$ \\
Moderada & $38(12,7)$ \\
Grave & $10(3,3)$ \\
Muito grave & $10(3,3)$ \\
\hline
\end{tabular}

$\mathrm{n}$ = frequência absoluta; \% = frequência percentual.

Teste qui-quadrado de Pearson.

Quanto ao perfil desta amostra, observou-se que, entre os estudantes com FS, a maioria se caracterizava com idade entre 18 e 25 anos (92,3\%; $n=85$ ), sexo feminino $(79,4 \% ; n=73)$, solteira $(94,5 \% ; n=87)$, cursando o primeiro ou segundo ano da faculdade $(67,4 \% ; n=62)$ e cursando uma universidade privada $(72,9 \% ; n=67)$ (Tabela 2).

Tabela 2 - Características epidemiológicas da população com transtorno de ansiedade social em Sergipe

\begin{tabular}{lcc}
\hline Característica & $\begin{array}{c}\text { Prevalência, } \\
\mathbf{n}(\%)\end{array}$ & p-valor \\
\hline 18-25 anos de idade & $85(92,3)$ & 0,897 \\
26-33 anos de idade & $5(5,4)$ & 0,897 \\
Acima de 33 anos de idade & $2(2,3)$ & 0,897 \\
Sexo feminino & $73(79,4)$ & 0,000 \\
Sexo masculino & $19(20,6)$ & 0,000 \\
Solteiro & $87(94,5)$ & 0,314 \\
Casado/união estável/separado & $5(5,5)$ & 0,314 \\
Instituição pública & $25(27,1)$ & 0,322 \\
Instituição privada & $67(72,9)$ & 0,322 \\
Primeiro e segundo ano do curso & $62(67,4)$ & 0,801 \\
de medicina & & \\
Terceiro e quarto ano do curso de & $30(32,6)$ & 0,801 \\
medicina & & \\
\hline
\end{tabular}

$\mathrm{n}$ = frequência absoluta; \% = frequência percentual. Teste qui-quadrado de Pearson.

Ademais fomos à busca dos principais medos e evitações enfrentados pelos universitários com FS. As principais situações sociais encontradas que cursam com 
Acadêmica de Medicina, Universidade Tiradentes, Aracaju, SE. 2 Acadêmico de Medicina, Universidade Tiradentes, Aracaju, SE. ${ }^{3}$ Graduado em Medicina, Universidade Federal de Sergipe (UFS), Lagarto, SE. ${ }^{4}$ Doutora. Professora, Universidade Tiradentes, Aracaju, SE. Professora, UFS, São Cristóvão, SE.

medo foram expressar discordância ou desaprovação para alguém (97,8\%) e falar com alguém que não conhece (95,7\%). Quando analisadas essas situações nos indivíduos sem FS, os medos sociais foram menos comuns. (Tabela 3).

Falar em público ao apresentar um seminário, por exemplo, foi uma situação social associada ao medo em $62,4 \%$ dos participantes sem fobia e em 94,6\% dos universitários com FS; assim como o medo de participar de pequenos grupos, como uma tutoria, relatado por 43,5\% dos estudantes sem FS, com uma prevalência de $75,2 \%$ no grupo com FS.
Com relação à prevalência de evitação das situações sociais, as mais frequentes foram expressar discordância ou desaprovação (98,9\%), falar com pessoas desconhecidas (95,7\%) e ser o centro das atenções (93,5\%). A evitação de situações sociais também foi analisada em estudantes sem o transtorno, o que permitiu a comparação (Tabela 4).

\section{DIsCUSSÃo}

No presente estudo, 30,7\% ( $n=92)$ dos estudantes de medicina foram diagnosticados como portadores de FS em alguma gravidade. Um percentual próximo a este foi encontrado no estudo de Regis ${ }^{16}$, no qual $36,3 \%$ dos

Tabela 3 - Prevalência dos diferentes medos nos indivíduos sem e com fobia social em Sergipe

\begin{tabular}{lccc}
\hline Medo & Sem FS, $\mathbf{n}(\%)$ & Com FS, $\mathbf{n}(\%)$ & p-valor \\
\hline Telefonar em público & $55(26,6)$ & $56(60,8)$ & $<0,001$ \\
Participar de pequenos grupos & $90(43,5)$ & $72(75,2)$ & $<0,001$ \\
Comer em locais públicos & $17(8,2)$ & $35(38)$ & $<0,001$ \\
Beber em locais públicos & $22(10,7)$ & $36(39)$ & $<0,001$ \\
Falar com pessoas em posição de autoridade & $133(64,2)$ & $87(94,5)$ & $<0,001$ \\
Apresentar um seminário & $130(62,4)$ & $87(94,6)$ & $<0,001$ \\
Ir a uma festa & $43(20,8)$ & $49(53,3)$ & $<0,001$ \\
Trabalhar sendo observado & $123(59,5)$ & $87(94,5)$ & $<0,001$ \\
Escrever sendo observado & $89(42,9)$ & $73(9,4)$ & $<0,001$ \\
Chamar alguém que não conhece & $114(55,1)$ & $85(92,4)$ & $<0,001$ \\
Falar com alguém que não conhece & $129(62,3)$ & $88(95,7)$ & $<0,001$ \\
Encontrar com estranhos & $126(60,9)$ & $87(94,6)$ & $<0,001$ \\
Urinar em público & $49(23,7)$ & $49(53,3)$ & $<0,001$ \\
Entrar em uma sala onde outros já estão sentados & $113(54,6)$ & $84(91,3)$ & $<0,001$ \\
Ser o centro das atenções & $148(71,5)$ & $87(94,6)$ & $<0,001$ \\
Falar em uma reunião & $108(52,2)$ & $82(89,1)$ & $<0,001$ \\
Fazer uma prova & $134(64,7)$ & $85(92,4)$ & $<0,001$ \\
Expressar discordância ou desaprovação & $132(63,8)$ & $90(97,8)$ & $<0,001$ \\
Olhar nos olhos de pessoa que você não conheça bem & $96(46,4)$ & $48(79,3)$ & $<0,001$ \\
Relatar algo para um grupo & $89(43)$ & $81(88)$ & $<0,001$ \\
Tentar impressionar alguém do sexo oposto & $108(52,2)$ & $85(92,4)$ & $<0,001$ \\
Devolver mercadorias em uma loja & $77(37,2)$ & $69(75)$ & $<0,001$ \\
Organizar uma festa normal & $78(37,7)$ & $76(82,6)$ & $<0,001$ \\
Resistir a um vendedor persistente & $49(23,7)$ & $69(75)$ & $<0,001$ \\
\hline FS & & &
\end{tabular}

FS = fobia social; $n$ = frequência absoluta; \% = frequência percentual.

Teste qui-quadrado de Pearson. 


\section{ARTIGO ORIGINAL}

\section{MAYANA LULA ANDRADE \\ KAMILA MARIA DE ANDRADE SANTOS SILVEIRA EUSÉBIO LINO DOS SANTOS JÚNIOR PAULO MILHOMEM FERRO NETO DEBORAH PIMENTEL}

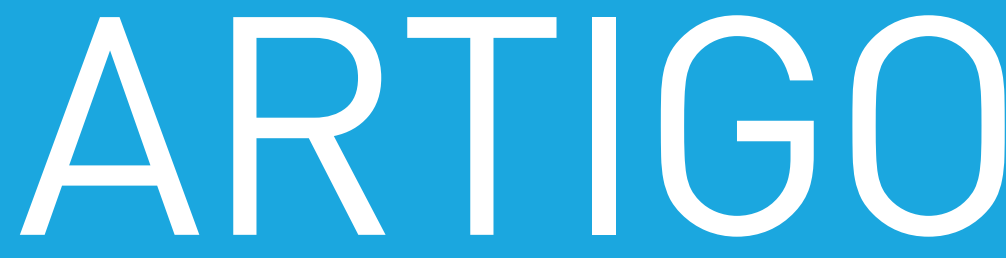

Tabela 4 - Prevalência das diferentes evitações nos indivíduos sem e com fobia social em Sergipe

\begin{tabular}{lccc}
\hline Evitação & Sem FS, $\mathbf{n}(\%)$ & Com FS, $\mathbf{n}(\%)$ & $\mathbf{p}$-valor \\
\hline Telefonar em público & $109(52,7)$ & $71(77,2)$ & $<0,001$ \\
Participar de pequenos grupos & $62(30)$ & $60(65,2)$ & $<0,001$ \\
Comer em locais públicos & $34(20,8)$ & $41(44,6)$ & $<0,001$ \\
Beber em locais públicos & $43(20,8)$ & $47(51,1)$ & $<0,001$ \\
Falar com pessoas em posição de autoridade & $118(57)$ & $83(90,2)$ & $<0,001$ \\
Apresentar um seminário & $109(52,7)$ & $79(85,9)$ & $<0,001$ \\
Ir a uma festa & $65(31,4)$ & $57(62)$ & $<0,001$ \\
Trabalhar sendo observado & $90(43,5)$ & $79(85,9)$ & $<0,001$ \\
Escrever sendo observado & $79(38,2)$ & $71(77,2)$ & $<0,001$ \\
Chamar alguém que não conhece & $113(54,6)$ & $85(92,4)$ & $<0,001$ \\
Falar com alguém que não conhece & $125(60,4)$ & $88(95,7)$ & $<0,001$ \\
Encontrar com estranhos & $122(58,9)$ & $85(92,4)$ & $<0,001$ \\
Urinar em público & $70(33,8)$ & $56(60,9)$ & $<0,001$ \\
Entrar em uma sala onde outros já estão sentados & $91(44)$ & $80(87)$ & $<0,001$ \\
Ser o centro das atenções & $152(73,4)$ & $86(93,5)$ & $<0,001$ \\
Falar em uma reunião & $91(44)$ & $75(81,5)$ & $<0,001$ \\
Fazer uma prova & $43(20,8)$ & $51(55,4)$ & $<0,001$ \\
Expressar discordância ou desaprovação & $133(64,2)$ & $91(98,9)$ & $<0,001$ \\
Olhar nos olhos de pessoa que você não conheça bem & $99(47,8)$ & $69(75)$ & $<0,001$ \\
Relatar algo para um grupo & $76(36,7)$ & $79(85,9)$ & $<0,001$ \\
Tentar impressionar alguém do sexo oposto & $101(48,8)$ & $79(85,9)$ & $<0,001$ \\
Devolver mercadorias em uma loja & $60(33,8)$ & $66(71,7)$ & $<0,001$ \\
Organizar uma festa normal & $80(38,6)$ & $68(73,9)$ & $<0,001$ \\
Resistir a um vendedor persistente & $58(28)$ & $68(73,9)$ & $<0,001$ \\
\hline FS fobia sol & &
\end{tabular}

FS = fobia social; $n$ = frequência absoluta; \% = frequência percentual.

Teste qui-quadrado de Pearson.

estudantes de medicina de uma universidade no estado de São Paulo apresentavam sintomas de FS. O estudo de Santos Filho et al. ${ }^{20}$, por sua vez, avaliou 355 estudantes universitários brasileiros e encontrou um percentual de 39,72\% ( $n=141)$ de alunos com o transtorno. Essa prevalência aumentada da FS, segundo Gavioliin, pode ser explicada por vulnerabilidades sociodemográficas, como renda familiar, moradia, extensa grade curricular e sobrecarga de atividades que levam ao estresse, redução do desempenho acadêmico e da qualidade de vida.

Santos Filho et al. ${ }^{20}$ demonstraram também que a FS moderada prevalece $(25,91 \%)$, o que corrobora os resultados encontrados por Gavioli21, em que, dos 21\% de alunos com FS, 10\% representavam a FS moderada; e com os de Meotti \& Mahl ${ }^{22}$, em que a FS moderada foi a de maior percentual (55,9\%). Nosso estudo, portanto, confirma esse predomínio de FS moderada, com o valor de $12,7 \%$. $O$ estudo de Lindo et al. ${ }^{23}$, entretanto, foi discordante e mostrou que a maioria dos estudantes tinha FS leve $(77,4 \%$,), em comparação a 20,5\% da moderada e 2,1\% da FS grave.

Outros trabalhosapresentam uma porcentagem inferior aos dados supracitados, como o estudo de Baptista ${ }^{17}$, que selecionou, aleatoriamente, 2.319 estudantes em duas universidades brasileiras e evidenciou uma prevalência de $11,6 \%$ de FS ( $n=237)$. 


\section{MAYANA LULA ANDRADE', KAMILA MARIA DE ANDRADE SANTOS SILVEIRA', EUSÉBIO LINO DOS SANTOS JÚNIOR², PAULO MILHOMEM FERRO NETO³, DEBORAH PIMENTEL ${ }^{4}$}

Acadêmica de Medicina, Universidade Tiradentes, Aracaju, SE. ${ }^{2}$ Acadêmico de Medicina Universidade Tiradentes, Aracaju, SE. ${ }^{3}$ Graduado em Medicina, Universidade Federal de Sergipe (UFS), Lagarto, SE. ${ }^{4}$ Doutora. Professora, Universidade Tiradentes, Aracaju, SE. Professora, UFS, São Cristóvão, SE.

Ademais, o estudo de Tillfors \& Furmark ${ }^{24}$ detectou um percentual de $16,1 \%$ de estudantes com FS. É válido dizer que, mesmo com as diferenças percentuais encontradas, a FS é um dos transtornos mentais de maior prevalência no meio acadêmico ${ }^{8,25}$ As divergências na literatura podem ser explicadas pelo fato de as pesquisas não se concentrarem em investigar especificamente os estudantes de medicina, população mais propensa a desenvolver o transtorno.

Além disso, a alta prevalência de FS em nossa amostra pode ser em decorrência da metodologia de ensino aplicada nesses universitários. Ou seja, o método ABP pode representar um gatilho para sintomas ansiosos, uma vez que essa metodologia exige maior participação ativa do estudante no seu processo de aprendizagem ${ }^{14}$. Não obstante, o gerenciamento e a construção do seu próprio aprendizado pode gerar no aluno um sentimento de incapacidade diante de tamanha responsabilidade ${ }^{14,26}$. Ademais, nesta metodologia, o estudante é analisado diariamente quanto ao seu desempenho, habilidade, atitude, senso crítico e capacidade de autoavaliação, favorecendo o agravamento dos sintomas da FS ${ }^{14}$.

Sabe-se que a população feminina apresenta maiores índices de FS quando comparada à masculina, chegando a uma proporção de 1,4:1, respectivamente $3,11,27,28$. Uma maior prevalência no sexo feminino também foi encontrada no estudo atual: entre os indivíduos com FS, $79,4 \%$ são mulheres, enquanto $20,6 \%$ são homens. Entretanto, Wagner et al. ${ }^{8}$, em um estudo com 87 mulheres de uma universidade no Rio Grande do Sul, encontraram um percentual abaixo do esperado, de 35,6\% de universitárias com FS.

Uma proporção elevada de FS no sexo feminino também foi evidenciada por Kinrys \& Wygant ${ }^{29}$, que, por sua vez, atribuíram essas diferenças a interações genéticas, hormonais e ambientais relacionadas a dilemas na infância. Em contraste às diferenças aventadas acima, Caponi ${ }^{30}$ indica que o transtorno em questão não tem alteração em relação a sexo ou raça/etnia.

Conforme a nossa amostra, índices maiores de FS foram encontrados nos alunos do primeiro e segundo anos em comparação aos alunos do terceiro e quarto anos, correspondendo a 67,4 e 32,6\%, respectivamente. Bento et al. ${ }^{14}$ descrevem que a FS costuma ser mais frequente no início do curso, devido ao receio da não adaptação e medo da constante avaliação dos colegas e professores. O estudo de Nascimento ${ }^{27}$, o qual utilizou a mesma escala para FS que a nossa pesquisa, também encontrou uma prevalência da FS em 60\% dos universitários que estão iniciando a graduação e 40\% naqueles que já estão no fim do curso. Por outro lado, os estudos de Carneiro \& Teixeira ${ }^{31}$ e o de Del Prette et al. ${ }^{32}$ não descrevem diferenças na prevalência de FS em relação ao ano em que o estudante se encontra.

Com relação ao estado civil, a grande maioria dos indivíduos com FS estava solteira, correspondendo a $94,5 \%$ ( $n=87)$. Há de se dizer que a população estudada possui, na sua maior parte, pessoas entre 18 e 25 anos, na qual a pouca idade pode explicar o fato de a maioria estar solteira. Outrossim, é sabido que a preponderância de FS em indivíduos solteiros se deve ao fato de que estes apresentam dificuldade de estabelecer uma relação afetiva estável, devido ao seu comprometimento de interação social ${ }^{8,24,27}$.

Quando estudadas as situações sociais mais temidas nas pessoas com FS, destacam-se as dificuldades associadas a atividades educacionais, tais como falar em público, tirar dúvidas e participar de atividades extracurriculares e eventos sociais ${ }^{33}$. Além do medo da possibilidade de cometer um erro, parecer pouco atrativo ou incompetente, as pessoas com FS frequentemente têm medo de exibir sintomas que possam ser interpretados como sinais de ansiedade. Como consequência de uma experiência prévia interagindo com predisposições comportamentais, fóbicos sociais desenvolvem uma série de suposições sobre eles próprios e sua rede social, o que thes fazem acreditar que estariam em perigo em uma ou mais situações sociais e, assim, acabam com medo. Eles mantêm essa preocupação não só porque assumem que outras pessoas irão notar esses sintomas, mas também porque outros podem usá-los para tirar conclusões caracterológicas sobre eles ${ }^{34}$.

Em nossa pesquisa, os alunos com FS mostraram uma maior prevalência de cada um dos medos e evitações avaliados em comparação aos sujeitos sem o transtorno. O maior medo foi o de expressar discordância e desaprovação, tanto em indivíduos saudáveis (63,8\%) como naqueles que apresentaram sintomas de FS (97,8\%). Esse achado foi compatível com o estudo de Bandeira ${ }^{35}$, em que a categoria "expressar insatisfação 


\section{ARTIGO ORIGINAL}

\section{MAYANA LULA ANDRADE \\ KAMILA MARIA DE ANDRADE SANTOS SILVEIRA EUSÉBIO LINO DOS SANTOS JÚNIOR PAULO MILHOMEM FERRO NETO DEBORAH PIMENTEL}

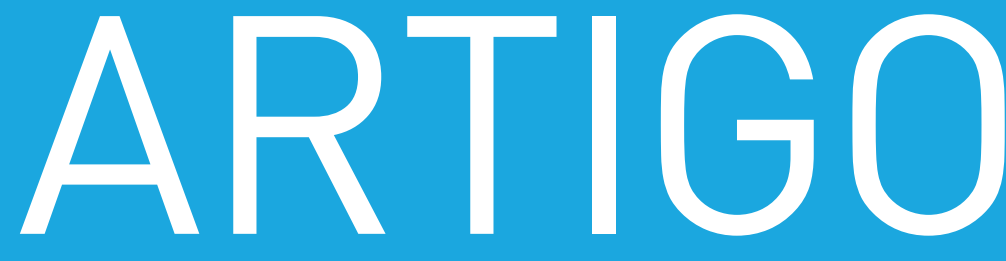

e/ou solicitar mudança de comportamento" foi citada como a mais desagradável por universitários.

Nossos resultados também mostram o medo de falar em público como um dos sintomas de maior prevalência. Entre os alunos com FS, 94,6\% relataram medo ao apresentar um seminário e 89,1\% ao falar em uma reunião. Muitos estudos trazem esse resultado como o mais comum, tanto em sujeitos saudáveis quanto nos que têm FS $8,17,36-38$. Essa situação pode ser exemplificada por D'El Rey \& Pacini ${ }^{38}$, que trouxeram a prevalência de $32 \%$ de medo, nervosismo ou desconforto em falar público em amostras da população geral. Corroborando, ainda, com estas informações, o estudo brasileiro de Baptista ${ }^{28}$ analisou os tipos de medos presentes nos sujeitos com FS, e o mais prevalente foi também o de falar em público (91,6\%).

Deve-se ressaltar que falar em público é uma atividade acadêmica comum, principalmente quando o método de ensino é a ABP. Assim, a FS associa-se a um desempenho acadêmico prejudicado, ou seja, está ligada a um desempenho educacional menor. A pesquisa de D'El Rey \& Pacini ${ }^{38}$ mostrou que $13 \%$ dos entrevistados relataram que o medo de falar em público resultou em grande interferência em seu trabalho, vida social e educação ou causou sofrimento acentuado. Outros estudos com indivíduos com FS no contexto universitário apontam que a situação que mais gerou ansiedade em até 71,4\% dos alunos é falar em frente a um grupo, prejudicando o seu rendimento acadêmico ${ }^{24,30}$.

Outro dado encontrado em Baptista ${ }^{28}$ foi o de que, após falar em público, a segunda situação social mais relatada foi comer em público (28,3\%), seguida de escrever em público (16,5\%) - estas duas últimas situações tiveram uma menor importância em nosso estudo, quando comparadas aos demais itens (38 e 9,4\%, respectivamente).

Em um estudo indiano feito por Shah \& Kataria ${ }^{25}$, são abordados os medos de urinar em banheiros públicos e de ser o centro das atenções, cada um presente em $20 \%$ dos estudantes entrevistados, em contraste com os valores da nossa pesquisa, em que 53,3 e 94,6\% dos participantes fóbicos apresentam esses sintomas, respectivamente.

Outra característica chave da FS é a evitação de determinadas situações sociais. Heiser et al. ${ }^{36}$ revelaram que quase todos os indivíduos com FS (96\%) relataram evitar algumas situações sociais das quais eles têm medo. As situações mais comumente evitadas foram conversações, falar em público, reuniões, aulas ou festas. Na nossa casuística, as mais frequentes evitações foram a de expressar discordância ou desaprovação (98,9\%), falar com pessoas desconhecidas (95,7\%) e ser o centro das atenções (93,5\%). Em contraste, Ranta et al. ${ }^{39}$ realizaram um estudo entre jovens com idades próximas aos da nossa amostra, e os resultados são destoantes: ser o centro das atenções foi um dos dois itens mais ditos como evitados pelos participantes (8,5\%), seguido por falar em público, com 28,4\%.

Uma das grandes limitações deste estudo foi não ter realizado um estudo comparativo entre a nossa amostra de alunos submetidos ao ABP e estudantes da metodologia tradicional no estado de Sergipe, o que aponta a necessidade de novos estudos complementares.

\section{CONCLUSÃO}

A FS teve uma alta prevalência entre a amostra de universitários do curso de medicina submetidos à metodologia de ensino ABP no estado de Sergipe, sendo que a maioria dos casos pertence à gravidade moderada e leve do transtorno. As situações sociais mais temidas e relacionadas com sintomas de medo e ansiedade foram expressar uma desaprovação ou discordância, seguida de falar com desconhecidos, apresentar seminários e ser o centro das atenções. Haja vista as repercussões negativas desse transtorno, com este trabalho colocamos o tema em evidência, servindo de base para novos estudos com uma amostra mais ampla, que incluam alunos de metodologia tradicional e possibilitem uma visão mais abrangente do problema na universidade.

\section{Agradecimentos}

Nosso agradecimento especial à Dra. Déborah Pimentel, parte fundamental na construção deste artigo. Agradecemos também aos alunos de medicina da Universidade Tiradentes e da Universidade Federal de Sergipe (Campus Lagarto), os quais de bom grado contribuíram com suas informações, indispensáveis para o desenvolvimento do projeto.

À Universidade Tiradentes, pelo incentivo aos jovens universitários às novas descobertas na área da pesquisa 


\section{MAYANA LULA ANDRADE' ${ }^{1}$ KAMILA MARIA DE ANDRADE SANTOS SILVEIRA', EUSÉBIO LINO DOS SANTOS JÚNIOR², PAULO MILHOMEM FERRO NETO³, DEBORAH PIMENTEL ${ }^{4}$}

Acadêmica de Medicina, Universidade Tiradentes, Aracaju, SE. ${ }^{2}$ Acadêmico de Medicina, Universidade Tiradentes, Aracaju, SE. ${ }^{3}$ Graduado em Medicina, Universidade Federal de Sergipe (UFS), Lagarto, SE. ${ }^{4}$ Doutora. Professora, Universidade Tiradentes, Aracaju, SE. Professora, UFS, São Cristóvão, SE.

através do Programa de Bolsas de Iniciação Científica (PROBIC/UNIT).

Artigo submetido em 23/07/2019, aceito em 01/10/2019. Os autores informam não haver conflitos de interesse associados à publicação deste artigo.

Fonte de financiamento: Esse estudo contou com o apoio financeiro do programa institucional de Bolsas de Iniciação Científica da Universidade Tiradentes de Aracaju-Sergipe PROBIC/Unit.

Correspondência: Mayana Lula Andrade, Av. Deputado Silvio Teixeira, 1283/504, Condomínio Delphinus, Bloco B, Bairro Jardins, CEP 49015-100, Aracaju, SE. E-mail: mayana.lula.andrade@gmail.com

\section{Referências}

1. Fernández-Sogorb A, Aparicio-Flores MP, Granados L, Aparisi-Sierra D, Inglés, CJ. Ansiedad social y fobia social: revisión de autoinformes y análisis de su frabilidad y validez en muestra infantojuvenil española. Calidad Vida Salud. 2018;11:30-6.

2. Buckner JD, Dean KE. Social anxiety and postevent processing among African-American individuals. Anxiety Stress Coping. 2017;30:21927.

3. Rudaz M, Ledermann T, Margraf J, Becker ES, Craske MG. The moderating role of avoidance behavior on anxiety over time: Is there a difference between social anxiety disorder and specific phobia? PLoS One. 2017;12:e0180298.

4. Taylor CT, Pearlstein SL, Stein MB. The affective tie that binds: examining the contribution of positive emotions and anxiety to relationship formation in social anxiety disorder. J Anxiety Disord. 2017:49:21-30.

5. Ballesteros F, Labrador FJ. Análisis de las técnicas psicológicas utilizadas en el trastorno de fobia social en un centro sanitario de psicología. Clinica y Salud. 2018; 29:71-80.

6. Mendanha ACT, Bernardes LA. Transtorno de ansiedade social e a não aceitação da homossexualidade: revisão narrativa. PUCMINAS. 2018;3:133-52.
7. Bas-Hoogendam JM, van Steenbergen $\mathrm{H}$, Nienke Pannekoek J, Fouche JP, Lochner C, Hattingh CJ, et al. Voxel-based morphometry multi-center mega-analysis of brain structure in social anxiety disorder. Neuroimage Clin. 2017;16:678-88.

8. Wagner MF, Wahl SD, Cecconello WW. Sintomas de fobia social no ensino superior: uma amostra de população feminina. Adv Health Psychol. 2014;22:49-54.

9. Pereira SM, Lourenço LM. O estudo bibliométrico dotranstorno deansiedadesocialemuniversitários. Arq Bras Psicol. 2012;64:47-62.

10. Clerkin EM, Sarfan LD, Parsons EM, Magee JC. Mindfulness facets, social anxiety, and drinking to cope with social anxiety: testing mediators of drinking problems. Mindfulness (N Y). 2017;8:15970.

11. Craske MG, Stein MB. Anxiety. Lancet. 2016:388:3048-59.

12. Pereira VM, Nardi AE, Silva AC. Sexual dysfunction, depression, and anxiety in young women according to relationship status: an online survey. Trends Psychiatry Psychother. 2013;35:55-61.

13. Wong N, Sarver DE, Beidel DC. Quality of life impairments among adults with social phobia: the impact of subtype. J Anxiety Disord. 2012;26:507.

14. Bento LM, de Andrade LP, Sales A, de Souza AP, de Souza AF, Batistona GT, et al. Percepção de alunos de medicina quanto à aprendizagem $X$ ansiedade na metodologia ativa. Rev Ens Educ Cienc Human. 2017;18:178-82.

15. Tenório LP, Argolo VA, de Sá HP, de Melo EV, de Oliva Costa EF. Saúde mental de estudantes de escolas médicas com diferentes modelos de ensino. Rev Bras Educ Med. 2016;40:574-82.

16. Regis JMO, Ramos-Cerqueira ATA, Lima MCP, Torres AR. Social anxiety symptoms and body image dissatisfaction in medical students: prevalence and correlates. J Bras Psiquiatr. 2018;67:65-73.

17. Baptista CA, Loureiro SR, de Lima Osório F, Zuardi AW, Magalhães PV, Kapczinski F, et al. Social phobia in Brazilian university students: prevalence, 
under-recognition and academic impairment in women. J Affect Disord. 2012;136:857-61.

18. Gil AC. Métodos e técnicas de pesquisa social. São Paulo: Atlas; 2008.

19. Liebowitz MR. Social phobia. Mod Probl Pharmacopsychiatry. 1987;22:141-73.

20. Filho AS, Hetem LA, Ferrari MC, Trzesniak C, Martín-Santos R, Borduqui $\mathrm{T}$, et al. Social anxiety disorder: what are we losing with the current diagnostic criteria? Acta Psychiatr Scand. 2010;121:216-26.

21. Gavioli BD. Vulnerabilidades sociodemográficas para transtornos de ansiedade social em estudantes de enfermagem de uma instituição de autarquia estadual [monografia]. São José do Rio Preto: FAMERP; 2017.

22. Meotti L, Mahl ÁC. Fobia Social: incidência em acadêmicos de psicologia. Unoesc Cienc ACBS. 2015;6:89-96.

23. Lindo L, Vega-Dienstmaier J, Lindo M, Cortés J. Prevalencia de síntomas de fobia social en adolescentes varones del Colegio Salesiano de Breña. Rev Neuropsiquiatr. 2005;68:228-40.

24. Tillfors M, Furmark T. Social phobia in Swedish university students: prevalence, subgroups and avoidant behavior. Soc Psychiatry Psychiatr Epidemiol. 2007:42:79-86.

25. Shah P, Kataria L. Social phobia and its impact in Indian university students. Internet J Ment Health. 2010;6:1-8.

26. Reis BMV, Donalonso JM, Pasquarelli A, Guizardi E, Vilela BP, Jaber MI, et al. O impacto da metodologia ativa de ensino na evolução dos sintomas de ansiedade social dentre os acadêmicos de medicina. Ensaios Cienc. 2013;17:31-47.

27. Nascimento BRC. Fobia Social: a incidência em estudantes universitários [Internet]. [cited Jun 2013]. psicologado.com.br/psicopatologia/ transtornos-psiquicos/fobia-social-a-incidenciaem-estudantes-universitarios.

28. Baptista, CA. Estudo da prevalência do transtorno de ansiedade social em estudantes universitários [dissertação]. Ribeirão Preto: Faculdade de Medicina, USP; 2006.

29. Kinrys G, Wygant LE. [Anxiety disorders in women: does gender matter to treatment?] Braz J Psychiatry. 2005;27:S43-50.

30. Caponi S. O DSM-V como dispositivo de segurança. Physis. 2014;24:741-63.

31. Carneiro AA, Teixeira CM. Avaliação de Habilidades Sociais em alunos de graduação em Psicologia da Universidade Federal do Maranhão. Psicol Ensino Form. 2011;2:43-56.

32. Del Prette ZAP, Del Prette A, Barreto MCM, Bandeira M, Rios-Saldaña MR, Ulian ALAO, et al. Social skills of psychology undergraduates: a multicentered study. Psicol Reflex Crit. 2004;17:341-50.

33. Van Ameringen M, Mancini C, Farvolden P. The impact of anxiety disorders on educational achievement. J Anxiety Disord. 2003;17:561-71.

34. Roth D, Antony, MM, Swinson RP. Interpretations for anxiety symptoms in social phobia. Behav Res Ther. 2001;39:129-38.

35. Bandeira M, Quaglia MAC. Habilidades sociais de estudantes universitários: identificação de situações sociais significativas. Inter Psicol. 2005;9:44-55.

36. Heiser NA, Turner SM, Beidel DC, Roberson-Nay R. Differentiating social phobia from shyness. J Anxiety Disord. 2009;23:469-76.

37. Ruscio AM, Brown TA, Chiu WT, Sareen J, Stein $M B$, Kessler RC. Social fears and social phobia in the USA: results from the National Comorbidity Survey Replication. Psychol Med. 2008;38:1528.

38. D'el Rey GJF, Pacini CA. Medo de falar em público em uma amostra da população: prevalência, impacto no funcionamento pessoal e tratamento. Psic Teor Pesq. 2005;21:237-42.

39. Ranta K, Kaltiala-Heino R, Koivisto AM, Tuomisto MT, Pelkonen M, Marttunen M. Age and gender differences in social anxiety symptoms during adolescence: The Social Phobia Inventory (SPIN) as a measure. Psychiatry Res. 2007;153:261-70. 